\title{
Expanding indications for the extended endoscopic endonasal approach to hypothalamic gliomas: preliminary report
}

\author{
Matteo Zoli, M.D., ${ }^{1}$ Diego Mazzatenta, M.D., ${ }^{1}$ Adelaide Valluzzi, M.D., ${ }^{1}$ \\ Gianluca Marucci, M.D., Ph.D., ${ }^{2}$ Nicola Acciarri, M.D., ${ }^{1}$ Ernesto Pasquini, M.D., ${ }^{3}$ \\ and Giorgio Frank, M.D. ${ }^{1}$ \\ ${ }^{1}$ Department of Neurosurgery, Center of Surgery for Pituitary Tumors and Endoscopic Skull Base Surgery, \\ IRCCS Istituto delle Scienze Neurologiche, Bellaria Hospital, Bologna; ${ }^{2}$ Department of Biomedical and \\ NeuroMotor Sciences (DiBiNeM), Section of Pathology, Bellaria Hospital, Bologna; and ${ }^{3}$ ENT \\ Department, Azienda Unità Sanitaria Locale di Bologna, Italy
}

Object. In the last decade the indications for the endoscopic endonasal approach have been progressively expanded to include lesions that have not been traditionally considered amenable to resection through the transsphenoidal route. In this study, the authors analyze their experience with hypothalamic gliomas treated via the endoscopic endonasal approach.

Methods. Consecutive cases of hypothalamic gliomas treated since 2007 via an endoscopic endonasal approach were reviewed. Preoperative and postoperative neuroimaging as well as endocrinological, neurological, and visual symptoms were analyzed to assess the surgical outcome. Signs and symptoms of hypothalamic dysfunction including body mass index (BMI), memory, sleep-wake rhythm, and polyphagia were prospectively collected pre- and postoperatively to assess hypothalamic function. Quality of life was evaluated using the Katz scale.

Results. In the initial phase the endoscopic endonasal approach was adopted in 3 cases with a palliative intent, to obtain a biopsy sample or for debulking of the mass followed by radio- or chemotherapy. In 2 later cases it was successfully adopted to achieve gross-total tumor resection. Complications consisted of 2 postoperative CSF leaks, which required an endoscopic endonasal reintervention. Visual deficit improved in 3 cases and normalized in the other 2. Four patients developed diabetes insipidus, and 3 an anterior panhypopituitarism. All patients had a moderate increase in BMI. No patients presented with any other signs of hypothalamic damage, and their quality of life at follow-up is normal.

Conclusions. Despite the limitations of a short follow-up and small sample, the authors' early experience with the endoscopic endonasal approach has revealed it to be a direct, straightforward, and safe approach to third ventricle astrocytomas. It allowed the authors to perform tumor resection with the same microsurgical technique: dissecting the tumor with 2 hands, performing a central debulking, and controlling the bleeding with bipolar coagulation. The main limitations were represented by some anatomical conditions, such as the position of the chiasm and the anterior communicating artery complex and, finally, by the challenge of watertight plastic repair. To definitively evaluate the role of this approach in hypothalamic gliomas, a comparison with transcranial series would be necessary, but due to the rarity of these cases such a study is still lacking. The authors observed that more aggressive surgery is associated with a worse endocrinological outcome; thus they consider it to be an open question (in particular in prepubertal patients) whether radical removal is an advisable goal for hypothalamic gliomas.

(http://thejns.org/doi/abs/10.3171/2014.7.FOCUS14317)

\section{KeY WORDS • endoscopic endonasal surgery • pilocytic astrocytoma • hypothalamic glioma $\quad$ extended transplanum/transtuberculum approach • cerebrospinal fluid leak}

\footnotetext{
Abbreviations used in this paper: $\mathrm{ACTH}=$ adrenocorticotropic hormone; $\mathrm{BMI}=$ body mass index; $\mathrm{DI}=$ diabetes insipidus; $\mathrm{FSH}=$ follicle-stimulating hormone; FT4 = free thyroxine; $\mathrm{GH}=$ growth hormone; $\mathrm{LH}=$ luteinizing hormone; $\mathrm{PA}=$ pilocytic astrocytoma; PMA = pilomyxoid astrocytoma; SDS = standard deviation score; $\mathrm{TSH}=$ thyroid-stimulating hormone.
}

I $\mathrm{N}$ the last decade the indications for the endoscopic endonasal approach have been progressively expanded to include routine resection not only of intracranial extradural lesions such as pituitary adenomas, but also of intradural lesions such as meningiomas and craniopharyngiomas. $5,6,8,14,23,24,29$ There are few reports on the 
utilization of the endoscopic endonasal route for intraparenchymal tumors such as hypothalamic gliomas, which are traditionally approached through various transcranial routes (pterional subfrontal, transcortical, or transcallosal) or via other minimally invasive approaches such as the supraorbital eyebrow craniotomy. ${ }^{10-12,15,26-28,30,31}$ In the present study we report the evolution of our experience with the endoscopic endonasal approach to hypothalamic gliomas, underlining the advantages and limits of this route, and review the current literature on the subject.

\section{Patient Population Methods}

We included every case of hypothalamic glioma treated with an endonasal endoscopic approach since 2007 (the year of the first case). These cases were selected based on the preoperative MRI findings. To determine eligibility for the endoscopic endonasal approach, we evaluated not only the location and extension of the tumor but specifically the relationship among the chiasm, the pituitary stalk, and the tumor. Every patient underwent a complete series of endocrinological baseline tests preoperatively (serum prolactin, cortisol, thyroid-stimulating hormone [TSH], adrenocorticotropic hormone $[\mathrm{ACTH}]$, free thyroxine [FT4], growth hormone $[\mathrm{GH}]$, luteinizing hormone $[\mathrm{LH}]$, follicle-stimulating hormone $[\mathrm{FSH}]$, and gonadotropin), as well as ophthalmological and neurological evaluation, and possible signs of diabetes insipidus (DI) were also investigated. Compromise of hypothalamic function was carefully analyzed, including assessment of body mass index (BMI) (BMI standard deviation score [SDS] in children), sleep/awake rhythm deregulation, memory dysfunction, compulsive hyperphagia, and psychic or behavioral alterations. The goal of resection (simple biopsy, debulking, or radical resection) was prospectively stated in the preoperative assessment by the operating neurosurgeon. Endocrinological, neurological, and ophthalmological assessments as well as MRI studies were repeated 3 months after surgery and annually thereafter. Adjuvant therapy (chemo- and/or radiotherapy) was considered in every case. Quality of life was evaluated using the Katz index of independence in activities of daily life (Table 1), considering 4 levels of functional outcome..$^{22}$

\section{Surgical Technique}

The details of our technique for endonasal endoscopic surgery have been described elsewhere. ${ }^{4,27}$ Briefly, surgery was done with rod lens endoscopes (Hopkins II, Karl Storz; $4 \mathrm{~mm}$ in diameter, $18 \mathrm{~cm}$ in length, with $0^{\circ}$ scope) with a high-definition camera. The patient is placed in the semisitting position, with the thorax slightly elevated on the operating table. The laryngopharynx is packed with gauze to prevent blood and fluid passage in the upper respiratory tract. We routinely use neuronavigation (StealthStation S7, Medtronic). Neuronavigation was based on a CT angiogram that was processed through StealthMerge software (Medtronic). The middle turbinate of the narrowest nasal fossa is resected while the contralateral one is laterally displaced. This is fol-
TABLE 1: Four levels of functional outcome were considered*

\begin{tabular}{|c|c|}
\hline Level & Functional Outcome \\
\hline 1 & $\begin{array}{l}\text { complete autonomy in daily activities and in social and work/ } \\
\text { academic tasks }\end{array}$ \\
\hline 2 & $\begin{array}{l}\text { partial autonomy in daily activities and in social and work/ } \\
\text { academic tasks }\end{array}$ \\
\hline 3 & $\begin{array}{l}\text { occasional external support necessary for daily life, impossible } \\
\text { to fulfill any social and work/academic tasks; a semidepen- } \\
\text { dent condition }\end{array}$ \\
\hline 4 & $\begin{array}{l}\text { daily life absolutely dependent on continuous external support; } \\
\text { a condition of absolute dependence }\end{array}$ \\
\hline
\end{tabular}

* Adapted from Katz et al., Journal of the American Medical Association, 1963.

lowed by a posterior septostomy to work through both nostrils. A wide anterior sphenoidotomy and monolateral anterior and posterior ethmoidectomy are then performed to expose the posterior wall of the sphenoid. Based on intraoperative anatomical landmarks (i.e., optic-carotid recess) the bony floor of the sella turcica is identified and progressively removed with a high-speed diamond drill or a Kerrison rongeur (Fig. 1A). Bone removal is extended superiorly to the tuberculum sellae and planum sphenoidale (Fig. 1B). The intercavernous sinus is coagulated, and then the dura mater is open superiorly to expose the suprasellar cistern (Fig. 1C). For the intradural phase of the surgery, we fix the endoscope with a holder to adopt a "four-hands" technique.

For an intradural, intraarachnoidal lesion like hypothalamic glioma, opening of the arachnoidal layer is necessary. It is important to preserve the arachnoid covering the optic nerves and their tiny feeding vessels and the superior hypophyseal artery. We usually open the arachnoid over the visible portion of tumor. If biopsy sampling was the goal of the surgery, small fragments are removed with a Weil forceps and hemostasis is achieved with bipolar coagulation (Fig. 2). In case of more extended tumor removal, the resection is performed with a technique similar to microsurgical resection: bimanual careful dissection of the tumor from the surrounding structures, using a cleavage plane when possible, cauterizing and cutting tumor feeding vessels, performing central debulking with the ultrasonic aspirator and suction, and then progressive centrifugal tumor removal (Fig. 3). In this phase, angled and double-angled instruments are extremely useful to reach the tumor without injuring surrounding structures. A waterproof plastic repair is performed with the freeflap multilayer technique, adopting fascia lata and mucoperiosteum, or with the "gasket seal" technique. A single 8-cm Merocel (Merocel Corp.) is placed in both nostrils.

\section{Results}

This series includes 5 patients ( 3 males and 2 females) with a mean age of 32 years (range 13-44 years). Preoperative MRI studies are shown in Fig. 4. None of the patients had received any previous treatment. The most common presentation consisted of various constellations of visual 


\section{Endoscopic endonasal approach to hypothalamic gliomas}
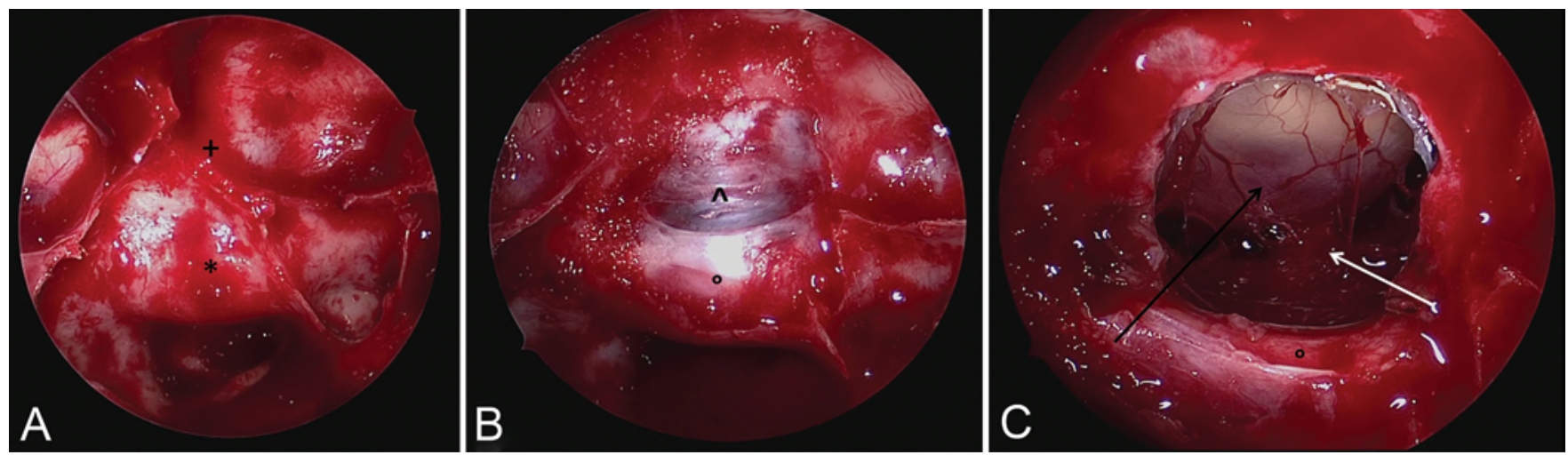

FIG. 1. Intraoperative view, $0^{\circ}$ angled scope. A: The sellar bulge is clearly visible at the posterior wall of the sphenoid sinus. Superiorly the tuberculum and planum sphenoidale region is exposed (*sellar bulge; +tuberculum sellae). B: After bone removal the dura of the pituitary gland is clearly visible as well as the dura of the suprasellar space $\left(^{\circ}\right.$ pituitary gland covered by dura; ^suprasellar dura). C: After opening of the dura, the suprasellar anatomy is recognizable and the tumor bulge in the hypothalamic region is shown ( ${ }^{\circ}$ pituitary gland; black arrow points to tumor mass; white arrow points to pituitary stalk).

complaints. Symptoms and signs at presentation are summarized in Table 2 . In the only patient of pediatric age (13 years old $)$, growth rate was normal $(155 \mathrm{~cm}, 50$ th percentile) and sexual maturation was not yet developed. The patient was overweight (60 kg, BMI SDS 2.1), but without an eating disorder. His academic status was normal. All of the adult patients had a normal social and working life. The mean BMI in the 4 adults was of $28.25 \mathrm{~kg} / \mathrm{m}^{2}$ (range $18-37$ $\mathrm{kg} / \mathrm{m}^{2}$ ). Two patients were obese, but none suffered eating disorders. No signs of hypothalamic dysfunction were present preoperatively in any of the 5 patients.

A simple biopsy procedure was performed in 2 cases in which there were mild visual symptoms with slow progression; an excisional biopsy to decrease mass effect was preferred in a patient with severe and progressive visual deterioration (right papillary atrophy and progressive worsening of the left visual acuity). In this latter case the tumor resection was extremely broad to decompress the optic nerves, tracts, and chiasm, and a left hypothalamic remnant was left because of its tenacious adhesion to the hypothalamus, the perforating vessels, and the carotid artery (Fig. 4B). In 2 cases a radical resection was performed. In both patients this was done because the preoperative working diagnosis was craniopharyngioma, the intraoperative histological diagnosis was nonspecific, and the presence of a good cleavage plane allowed a safe and complete dissection of the tumor from the surrounding parenchyma.

Surgical complications included a postoperative CSF leak in 2 patients that required endoscopic endonasal revision and graft replacement. In 4 cases the histological diagnosis was pilocytic astrocytoma (PA) (WHO Grade I) and in 1 it was pilomyxoid astrocytoma (PMA) (WHO Grade II) (Table 3). Pathological features are reported in Table 4. In the 4 cases of PA, microscopic examination showed a cellular, highly vascular tumor, composed of monomorphous, bipolar astrocytes, a biphasic growth pattern, Rosenthal fibers, and eosinophilic granular bodies. In the single case of PMA, neoplastic cells were arranged in a discohesive sheeting pattern, interspersed with microcytic spaces and myxoid matrix. The tumor cells were small to medium sized, with bipolar cytoplasmic processes arranged in an angiocentric fashion. Rosenthal fibers were absent and eosinophilic granular bodies were very rare.

With the exception of the 2 patients who underwent radical resection, each patient received adjuvant therapy, consisting of radiotherapy in 2 cases and an 18-month course of chemotherapy in the third. In both patients who underwent adjuvant radiotherapy, there was no further tu-
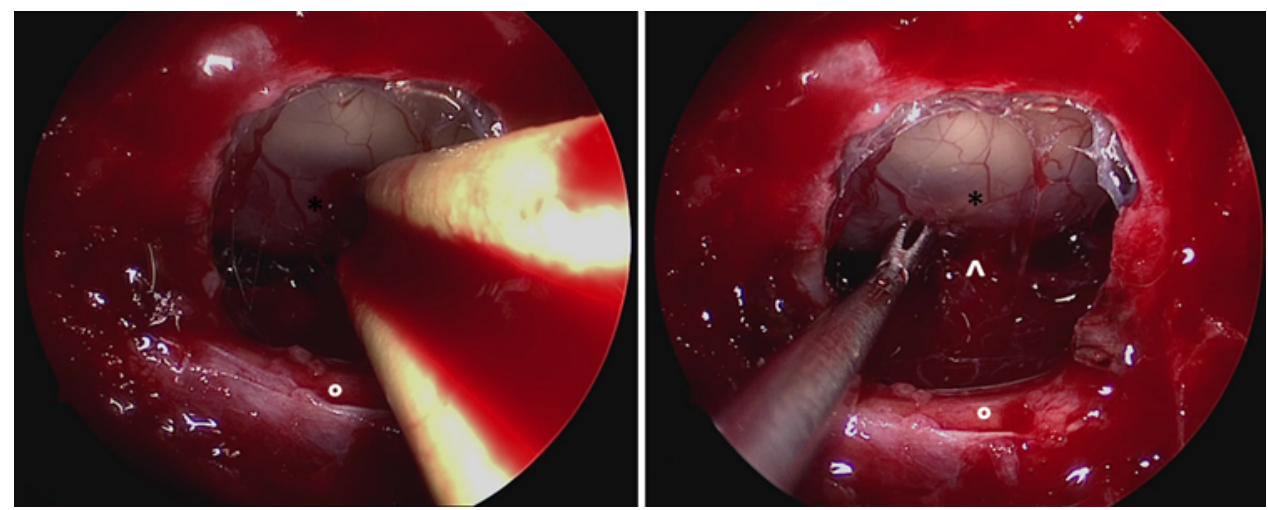

FIG. 2. Intraoperative view, $0^{\circ}$ angled scope. Left: Coagulation of the external surface of the tumor through a bipolar forcep. Right: Through an angled Weil forcep, a sample of the tumor is taken for biopsy ( ${ }^{\circ}$ pituitary gland; *tumor; ${ }^{\wedge}$ pituitary stalk). 

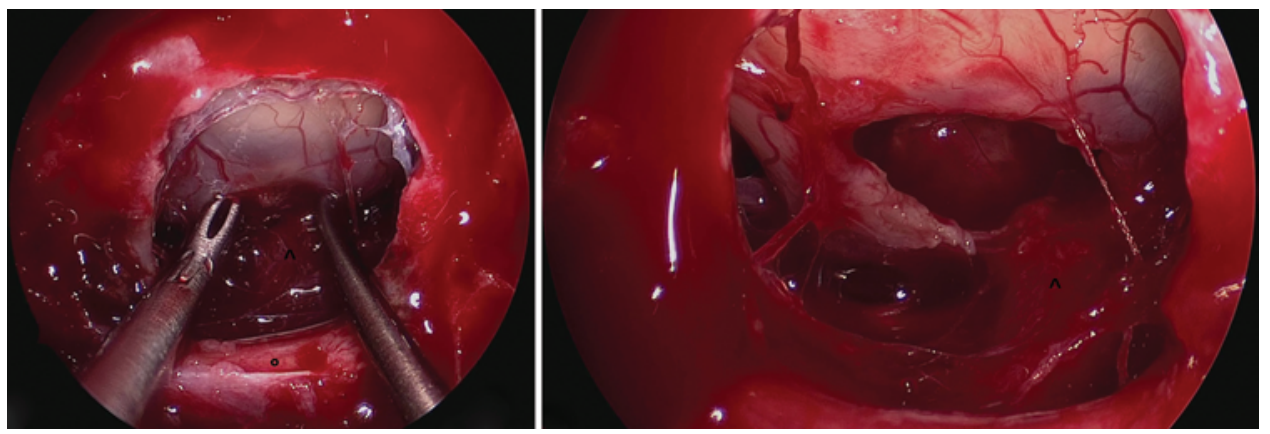

FIG. 3. Intraoperative view, $0^{\circ}$ angled scope. Left: Bimanual dissection of the tumor from the surrounding structure. Right: Final view after tumor removal. The stalk is preserved as well as the hypothalamic and chiasmatic feeding vessels. ${ }^{\circ}$ pituitary gland; ^ pituitary stalk.

mor growth after follow-up periods of 81 and 45 months, respectively (Fig. 4B and D). Conversely, after chemotherapy, the tumor had totally regressed at neuroradiological imaging at a follow-up of 28 months (Fig. 4F). Visual symptoms normalized in 2 patients and improved in the other 3. As summarized in Table 5, worse endocrinological outcomes were correlated with the aggressiveness of the resection (the more aggressive the resection, the worse the outcome), even though endocrinological function can deteriorate after a simple biopsy procedure, as in Case 2. The pediatric patient has continued normal growth $(178 \mathrm{~cm})$ and normal sexual maturation. However, after surgery he gained weight (88 kg, BMI SDS 2.3), even though no eating disorder was observed. In all adults an increase of the BMI was observed in the absence of frank eating disorders, and there were no sleep/wake rhythm or memory disturbances (Table 5). All patients returned to normal school and work activities and were independent in the activities of daily life. No recurrences were observed after a mean follow-up period of 35 months (range 3-81 months).

\section{Illustrative Case}

Case 4

History and Examination. A 38-year-old man with progressive visual loss over 1 year was referred to our institution with a working diagnosis of craniopharyngioma. Ophthalmological assessment revealed a bitemporal hemianopia and bilaterally reduced visual acuity $(6 / 10$ in the left eye and 8/10 in the right eye). The biohumoral assays revealed a deficit of the pituitary-adrenal axis (ACTH $30 \mathrm{pg} / \mathrm{ml}$, cortisol $24 \mathrm{ng} / \mathrm{ml}$ ), hypogonadotropic hypogonadism (LH $1.0 \mathrm{mIU} / \mathrm{ml}$, FSH $0.7 \mathrm{mIU} / \mathrm{ml}$, free testosterone $0.6 \mathrm{ng} / \mathrm{ml})$, and $\mathrm{GH}$ deficit $(\mathrm{GH}<0.05 \mathrm{ng} / \mathrm{ml})$. Other serum pituitary values were within normal limits. He had no history of polyuria or polydipsia, and was living a normal social and working life with no sign of hypothalamic dysfunction. At admission, his body weight was $78 \mathrm{~kg}$ and his height was $1.70 \mathrm{~m}\left(\mathrm{BMI} 27 \mathrm{~kg} / \mathrm{m}^{2}\right)$. A preoperative MRI study showed a suprasellar and mixed solid and cystic mass extending within the ventricle and displacing the optic chiasm anteriorly (Fig. 4G). The stalk was recognizable below the lesion. Our preoperative diagnosis was retrochiasmatic transpeduncular craniopharyngioma mainly involving the third ventricle.
Operation. Based on this working diagnosis, we opted for an endoscopic endonasal extended transplanum/ transtuberculum approach. Intraoperatively the lesion was found to be intradural and intraarachnoidal, and presented with a small anterior cyst in the suprasellar cistern; the cyst was drained and its wall resected. A biopsy sample of the posterior, solid portion of the tumor was obtained and the intraoperative histopathological diagnosis was chronic gliosis. The residual mass was friable, with a good cleavage plane from the surrounding structures; thus it was progressively removed. The stalk was recognizable posterior to the lesion, and was preserved. The intraoperative gross pattern of the tumor and the origin other than from the stalk was inconsistent with the hypothesis of craniopharyngioma. At the end, the tumor was radically removed, and the floor of the third ventricle was recognizable and free from neoplastic remnants. Plastic repair was performed with the free-flap multilayer technique, with fascia lata, bone, and mucoperiosteum put in place according to the "gasket seal" technique.

Postoperative Course. The postoperative course was marked by typical triphasic DI, and by a postoperative CSF leak that occurred 3 days after surgery and was promptly repaired. The patient experienced immediate postoperative improvement of his visual deficit. The final diagnosis was PA (WHO Grade I), and a follow-up MRI study obtained before discharge showed complete resection (Fig. 5). No adjuvant therapy was believed to be indicated. At a follow-up of 17 months there was normalization of his visual fields and of visual acuity. There was no improvement of his hypopituitarism, which required medical treatment, and he also had DI that was controlled with medical therapy. Follow-up MRI confirmed grosstotal resection with no evidence of residual or recurrent tumor (Fig. 4H). The patient experienced weight gain $(85$ $\mathrm{kg}$, BMI $29 \mathrm{~kg} / \mathrm{m}^{2}$ ), but no other hypothalamic sequelae were noted. He returned to his work 5 months after surgery, and at the last follow-up evaluation he reported returning to his presurgical quality of life.

\section{Discussion}

In this report we outline the evolution and results of the extended endoscopic endonasal approach in a selected group of patients with hypothalamic gliomas amenable 

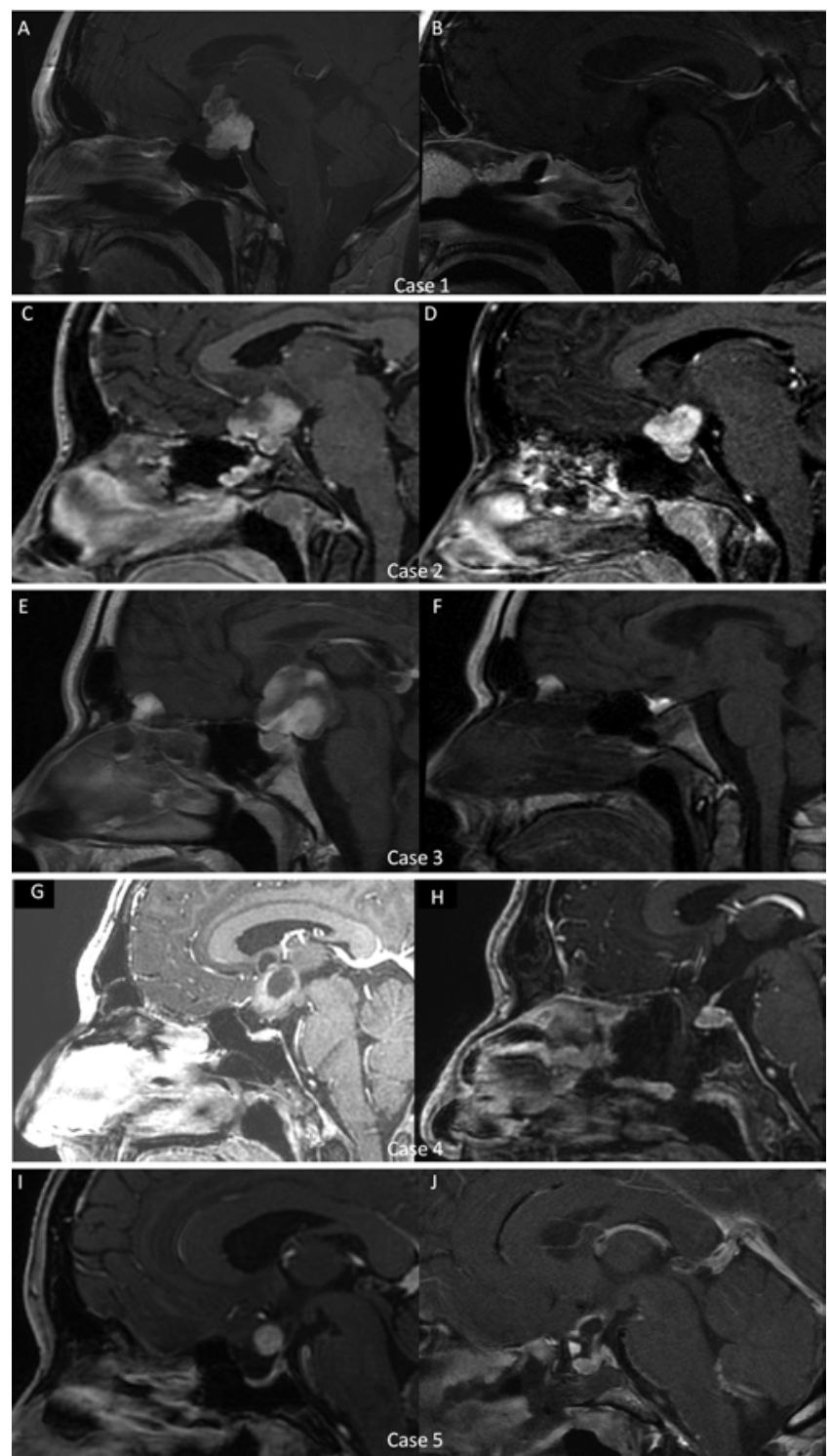

FIG. 4. Sagittal MRI studies obtained after administration of contrast. A and B: Case 1. Preoperative and postoperative (after 81 months) MRI showing the wide debulking of the mass. In panel $B$ the remnant of the tumor on the left side of hypothalamus is visible and remained stable at follow-up. C and D: Case 2. Preoperative and postoperative (after 45 months) MRI showing hypothalamic glioma, which remained stable at 45 months after biopsy and radiotherapy. E and F: Case 3. Preoperative and postoperative (after 18 months) MRI showing the regression of the tumor after biopsy and chemotherapy. $\quad \mathbf{G}$ and $\mathbf{H}$ : Case 4. Preoperative MRI study showing the tumor in the suprasellar region, involving the third ventricle $(G)$. The 2 portions (solid and cystic) are evident. Postoperative MRI study obtained 3 months after surgery showing that the gland and the stalk are preserved and visible, as are the mammillary bodies $(\mathrm{H})$. The tumor resection is radical. I and J: Case 5. Preoperative and postoperative (after 3 months) MRI showing the radical resection of the hypothalamic glioma.

to an approach from below, culminating with 2 patients in whom gross-total tumor removal was achieved. Initially, we adopted the endoscopic endonasal extended approach only for biopsy or debulking purposes. Moreover, in the past 2 years we have used an endonasal approach to treat 2 similar cases of hypothalamic gliomas originally suspected to be a craniopharyngioma, and we were able to achieve gross-total resection because the tumor was found to have a good cleavage plane from the surrounding structures. This suspicion arose because these last 2 cases were presenting some misleading neuroradiological features at neuroimaging. One case presented a mixed solid and cystic component, as in a craniopharyngioma; furthermore, in both cases no thickening or enhancing of optic nerves was present, and the origin of the tumor seemed to be from the pituitary stalk. Traditionally, tumors in the region of the third ventricle have been treated using a variety of different transcranial approaches (pterional subfrontal, transcortical, or transcallosal) or by using other minimally invasive approaches, such as the supraorbital eyebrow craniotomy. ${ }^{3,10-12,15,26-28,30,31}$ Recently, a few articles have reported the feasibility of an endoscopic endonasal approach to reach this region, ${ }^{8,9}$ in particular with regard to intradural extraarachnoidal lesions such as craniopharyngiomas. ${ }^{13,14}$

These observations and our previous experience with suprasellar craniopharyngiomas suggests that this same approach can be considered for selected patients with hypothalamic gliomas, with the goal of possible radical tumor removal, as our 2 last cases illustrated. The endoscopic transsphenoidal route was chosen because the tumor had created a corridor large enough to reach the hypothalamic region directly without any brain retraction, parenchymal incision, or vascular manipulation. ${ }^{9}$ Furthermore, this approach has been preferred to another minimally invasive approach such as the supraorbital eyebrow, because for cases with anterior or superior displacement of chiasm such as the ones included in this series, the endoscopic endonasal approach does not require opening of the lamina terminalis, reduces the risk of damage to optic nerves or chiasm, and provides a route that follows the tumor growth direction..$^{10,12,28,30}$

In our opinion, this point (i.e., the position of the chiasm in relation to the tumor and the tuberculum) is of pivotal importance to evaluate the feasibility of an endoscopic endonasal approach to the third ventricle. If the chiasm is pushed anteriorly and superiorly by the tumor, it is possible to use a corridor between the diaphragma sellae inferiorly, the inferior border of the chiasm superiorly, and the pituitary stalk posteriorly. This route is wide enough both to perform a simple biopsy of the tumor and to resect it partially or totally (Fig. 6 left). Conversely, if the chiasm is interposed along the surgical trajectory between the tuberculum and the tumor, this route cannot be adopted, because the resulting corridor would be too narrow to maneuver the instruments safely (Fig. 6 right). Indeed, even though in some studies an endoscopic endonasal route above the chiasm has been suggested, we considered this approach to be a major risk for visual chiasm or nerve damage. ${ }^{1}$ Therefore, careful analysis of high-resolution preoperative imaging studies is of paramount importance before this route is considered.

In all of the cases presented, the tumor itself, displacing the chiasm and the anterior communicating artery complex superiorly, created a favorable condition for an endoscopic endonasal approach. The endoscopic endonasal approach allows the surgeon to adopt a tumor removal 


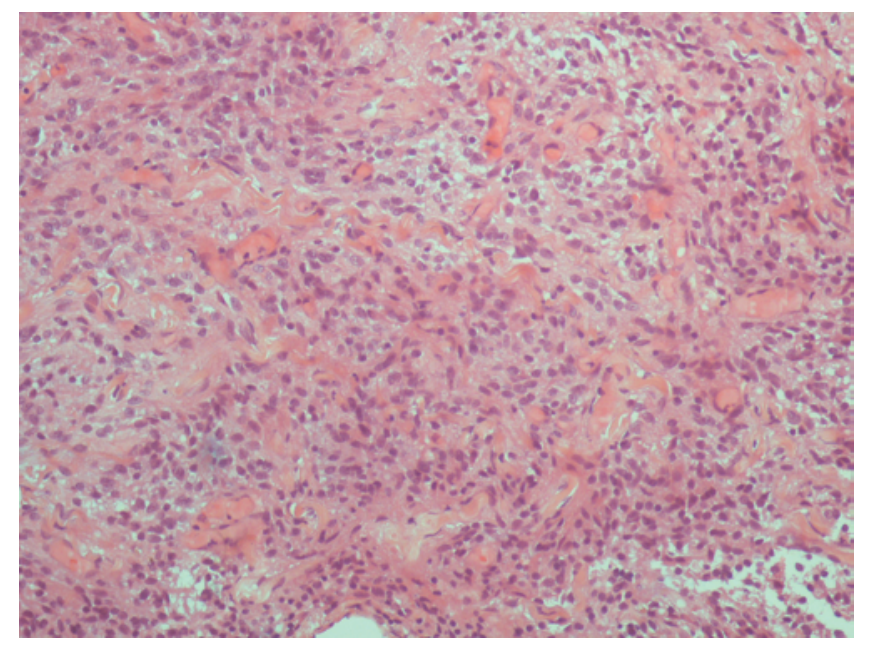

FIG. 5. Photomicrograph of tissue sample used in the histological examination. Microscopic examination showed a cellular, highly vascular tumor composed of monomorphous, bipolar astrocytes, a biphasic growth pattern, Rosenthal fibers, and eosinophilic granular bodies. H \& $\mathrm{E}$, original magnification $\times 100$.

technique not different from the microsurgical. The surgeon can work bimanually, dissecting the tumor from the surrounding structures, and performing a progressive debulking even with the use of the ultrasonic aspirator (Cavitron, Lasersonic Corp.). ${ }^{16-20}$ Furthermore, as in traditional microsurgical techniques, bleeding can be controlled with bipolar cautery or with hemostatic agents such as Floseal (Baxter). For removal of the tumor, angled instruments are extremely useful, allowing the surgeon to reach peripheral portions of the surgical field. One of the limits of this approach is represented by the narrowness of the surgical field, which can be partially resolved by performing an anterior and posterior ethmoidectomy to increase the maneuverability of the instruments and of the optics.

Our experience must be considered preliminary, and several considerations are in order. First, we ventured into the endoscopic endonasal partial and then total removal of hypothalamic gliomas only after several years of experience with this technique. Despite our extensive experience, in 2 of the 5 patients discussed, postoperative CSF leaks occurred and this complication remains one of the main limitations of the endoscopic endonasal approach applied to tumors of the third ventricle. Indeed, in these cases, after tumor removal the whole ventricular system is opened to the suprasellar cisterns, and the plastic repair
TABLE 3: Treatment and adjuvant therapies in 5 patients with hypothalamic gliomas

\begin{tabular}{clcl}
\hline Case No. & Aim of Surgery & $\begin{array}{c}\text { Histological } \\
\text { Finding }\end{array}$ & $\begin{array}{c}\text { Adjuvant } \\
\text { Treatment }\end{array}$ \\
\hline 1 & debulking & PA & radiotherapy \\
2 & biopsy & PA & radiotherapy \\
3 & biopsy & PA & chemotherapy \\
4 & radical resection & PA & none \\
5 & radical resection & PMA & none \\
\hline
\end{tabular}

should be resistant to high CSF pressure. .,21 $^{2}$ The size of the bone and dura opening is relevant for postoperative CSF leakage. In this setting, the risk of a postoperative CSF leak is increased by coughing and vomiting after emergence from anesthesia.? Thus, special care would be taken to have a smooth arousal from anesthesia, minimizing the effects of these maneuvers. Furthermore, careful observation and patient monitoring should be performed in the first days after surgery to detect any CSF leak early, avoiding the development of meningitis. Our choice to routinely perform the plastic repair with the free-flap multilayer and/or "gasket seal" technique, not adopting the nasoseptal flap, is aimed to avoid postoperative morbidity due to the flap, which requires frequent nasal medications, and can provoke nasal crusting, bleeding, and patient discomfort for weeks or months. ${ }^{2}$

Visual outcome was satisfactory in our series; all patients had an improvement or normalization of visual function. In our experience, to avoid postoperative visual deficits it is of paramount importance to preserve the feeding vessels of the chiasm, and in particular of the superior hypophyseal artery, as shown in Fig. 3. A further key point is the infiltration of the tumor into the optic apparatus. In the 2 cases in which a radical resection was achieved, a clear cleavage plane without tumoral infiltration of the chiasm was present, and indeed a good visual outcome was observed. Conversely, in case of observation of optic nerve or chiasm infiltration, the tumor removal should be halted.

As expected, endocrinological sequelae are more severe in patients undergoing more aggressive surgical treatment, and this must be weighted in the preoperative planning, especially when dealing with prepubertal patients, to avoid growth arrest or infertility. Thus, for these cases a less aggressive surgical treatment followed by ad-

TABLE 2: Preoperative symptoms and BMI in 5 patients with hypothalamic gliomas*

\begin{tabular}{ccllc}
\hline Case No. & Age (yrs), Sex & \multicolumn{1}{c}{ Visual Sx } & Endocrine Sx & BMI \\
\hline 1 & $42, \mathrm{M}$ & bilat acuity deficit, HH & none & 31 \\
2 & $23, \mathrm{~F}$ & bilat acuity deficit, incomplete BTH & none & 18 \\
3 & $13, \mathrm{M}$ & bilat acuity deficit, incomplete BTH & none & $2.1 \dagger$ \\
4 & $38, \mathrm{M}$ & bilat acuity deficit, complete BTH & partial ant hypopit & 27 \\
5 & $44, \mathrm{~F}$ & bilat acuity deficit, complete BTH & none & 37 \\
\hline
\end{tabular}

* ant = anterior; $\mathrm{BTH}=$ bitemporal hemianopia; $\mathrm{HH}=$ homonymous hemianopia; hypopit = hypopituitarism; $\mathrm{Sx}=$ symptoms.

$\dagger$ In the pediatric patient BMI was expressed as SDS BMI. 


\section{Endoscopic endonasal approach to hypothalamic gliomas}

TABLE 4: Pathological features of tumors in 5 patients with hypothalamic gliomas*

\begin{tabular}{cccccc}
\hline Case No. & Vascular Component & Macrophages & Hemosiderin & Calcifications & Diagnosis \\
\hline 1 & +++ & + & ++ & + & PA \\
2 & +++ & - & - & + & PA \\
3 & +++ & - & - & - & PA \\
4 & ++ & + & + & + & PA \\
5 & +++ & ++ & + & PMA \\
\hline
\end{tabular}

* + = scattered; ++ = common; +++ = prominent; - = absent.
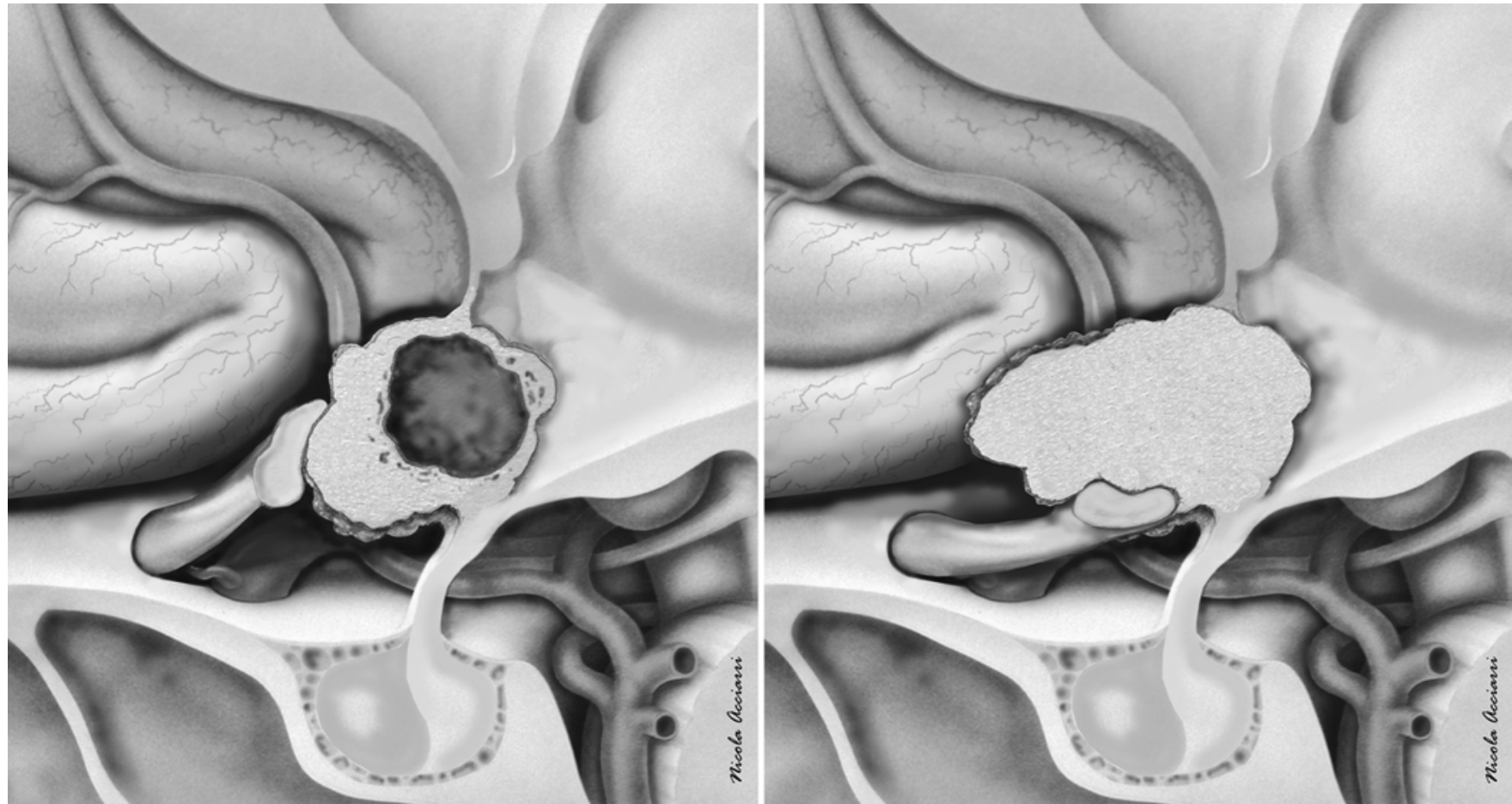

FIG. 6. Schematic drawings. Left: The chiasm is superiorly and anteriorly displaced by the tumor. A wide and direct corridor between the tuberculum sellae and the lesion can be followed to reach the tumor. Right: The chiasm is inferiorly pushed toward the tuberculum by the tumor. In this condition the endoscopic endonasal approach is not favorable to reach the tumor safely, because the chiasm is interposed in the surgical trajectory. Copyright Nicola Acciarri. Published with permission.

TABLE 5: Clinical outcome and quality of life in 5 patients with hypothalamic gliomas*

\begin{tabular}{cllcc}
\hline Case No. & Visual Outcome & Endocrinological Outcome & BMI & Level of Functional Outcome† \\
\hline 1 & improved & ant panhypopit \& DI & 32 & 1 \\
2 & normalized & DI & 19 & 1 \\
3 & improved & none & $2.3 \ddagger$ & 1 \\
4 & normalized & ant panhypopit \& DI & 29 & 1 \\
5 & improved & ant panhypopit \& DI & 39 & 1 \\
\hline
\end{tabular}

* panhypopit = panhypopituitarism.

$\dagger$ The functional outcome is expressed according to the modified Katz scale score presented in Table 1.

$\ddagger$ In the pediatric patient BMI was expressed as SDS BMI. 
juvant therapy can be the first choice. Conversely, in postpubertal patients the indication for a debulking or radical removal should be a balance between endocrinological morbidity, its related reduction of quality of life, and the severity of visual compromise. A postoperative weight gain occurred in all of our patients, independently from the surgical strategy. Other symptoms of hypothalamic derangement (memory compromise, sleep/wake cycle dysregulation, modification in behavior) did not occur in any of our patients. This experience is too limited to assess whether the endoscopic endonasal approach may be associated with a lower incidence of major hypothalamic compromise than other transcranial routes to these tumors.

We agree with other authors that the mammillary bodies are the posterior limit of any surgical corridor to the third ventricle and that absolute attention to sparing hypothalamic vessels is crucial to avoid irreversible consequences. ${ }^{25}$ We believe that the preservation of the hypothalamus and of its functions in hypothalamic gliomas depends also on the tumor architecture. Indeed, tumors with compact cellularity, which mainly displaces the surrounding nerve structures, can be removed with a better preservation of hypothalamic nuclei and fasciculi than can diffusely infiltrating forms. Therefore, careful preoperative planning and excellent surgical judgment and experience are required to find that delicate balance between aggressive tumor resection and preservation of function. Even if the great majority of gliomas of these regions have high cellularity and therefore compact morphology, functional MRI studies such as tractography can be useful to distinguish between compact tumor, (displacing the hypothalamic fasciculi) and dispersive tumors, (infiltrating and not displacing the fasciculi).

\section{Conclusions}

We report the evolution of the extended endoscopic endonasal approach to selected patients with hypothalamic astrocytomas treated by an approach from below. This initial experience may suggest that, in selected cases, the endoscopic endonasal approach allows the surgeon to remove the tumor with a microsurgical technique as well. This route requires particular anatomical conditions: the chiasm and anterior communicating artery should not be interposed along the surgical trajectory between the surgeon and the target. The endoscopic endonasal approach permits dissection and resection of the tumor while avoiding any brain manipulation or retraction. Cerebrospinal fluid leakage remains the main limitation of this surgery.

Our series has some limitations related to the relative rarity of these tumors, hence the small number of patients included. The follow-up is relatively short and the number of patients too small to make definitive conclusions regarding long-term results. Nevertheless, the preliminary experience from our group with this approach outlines possible future areas of investigation in our quest for less invasive techniques for complex midline intracranial tumors. In particular, in the case of an intraoperative finding of an unexpected intraparenchymal lesion, while performing an endoscopic extended approach for a pre- sumed craniopharyngioma, tumor removal can be technically possible. Considering that a more aggressive surgery is associated with worse endocrinological outcome, we think that it still is an open question whether radical removal is an advisable goal for hypothalamic gliomas, and it should be planned by evaluating the balance of endocrinological morbidity, related reduction of quality of life, and the severity of visual compromise.

\section{Acknowledgment}

We thank Dr. Nicola Acciarri for providing the artwork in Fig. 6.

\section{Disclosure}

The authors report no conflict of interest concerning the materials or methods used in this study or the findings specified in this paper.

Author contributions to the study and manuscript preparation include the following. Conception and design: Zoli. Acquisition of data: Zoli, Valluzzi, Marucci, Pasquini. Analysis and interpretation of data: Valluzzi, Marucci, Pasquini. Drafting the article: Zoli, Mazzatenta, Acciarri. Critically revising the article: Mazzatenta, Frank. Reviewed submitted version of manuscript: Frank. Administrative/technical/material support: Acciarri. Study supervision: Mazzatenta, Frank.

\section{References}

1. Abuzayed B, Tanriover N, Akar Z, Eraslan BS, Gazioglu N: Extended endoscopic endonasal approach to the suprasellar parachiasmatic cisterns: anatomic study. Childs Nerv Syst 26:1161-1170, 2010

2. Alobid I, Enseñat J, Mariño-Sánchez F, Rioja E, de Notaris M, Mullol J, et al: Expanded endonasal approach using vascularized septal flap reconstruction for skull base tumors has a negative impact on sinonasal symptoms and quality of life. Am J Rhinol Allergy 27:426-431, 2013

3. Apuzzo ML, Chikovani OK, Gott PS, Teng EL, Zee CS, Giannotta SL, et al: Transcallosal, interfornicial approaches for lesions affecting the third ventricle: surgical considerations and consequences. Neurosurgery 10:547-554, 1982

4. Apuzzo MLJ, Litofsky NS: Surgery in and around the anterior third ventricle, in Apuzzo MLJ (ed): Brain Surgery. New York: Churchill Livingstone, 1993, pp 541-580

5. Barazi SA, Pasquini E, D’Urso PI, Zoli M, Mazzatenta D, Sciarretta V, et al: Extended endoscopic transplanum-transtuberculum approach for pituitary adenomas. Br J Neurosurg 27:374-382, 2013

6. Cappabianca P, Cavallo LM, Esposito F, de Divitiis O, Messina A, de Divitiis E: Extended endoscopic endonasal approach to the midline skull base: the evolving role of transsphenoidal surgery. Adv Tech Stand Neurosurg 33:151-199, 2008

7. Castelnuovo P, Locatelli D, Mauri S, De Bernardi F: Extended endoscopic approaches to the skull base. Anterior cranial base CSF leaks, in de Divitiis E, Cappabianca P (eds): Endoscopic Endonasal Transsphenoidal Surgery. Vienna: Springer, 2003, pp 137-158

8. Cavallo LM, de Divitiis O, Aydin S, Messina A, Esposito F, Iaconetta G, et al: Extended endoscopic endonasal transsphenoidal approach to the suprasellar area: anatomic considerations - part 1. Neurosurgery 61 (3 Suppl):24-34, 2007

9. Cavallo LM, Solari D, Esposito F, Cappabianca P: The endoscopic endonasal approach for the management of craniopharyngiomas involving the third ventricle. Neurosurg Rev 36:27-38, 2013

10. Ditzel Filho LF, McLaughlin N, Bresson D, Solari D, Kassam AB, Kelly DF: Supraorbital eyebrow craniotomy for removal 


\section{Endoscopic endonasal approach to hypothalamic gliomas}

of intraaxial frontal brain tumors: a technical note. World Neurosurg 81:348-356, 2014

11. Fahlbusch R, Honegger J, Paulus W, Huk W, Buchfelder M: Surgical treatment of craniopharyngiomas: experience with 168 patients. J Neurosurg 90:237-250, 1999

12. Fatemi N, Dusick JR, de Paiva Neto MA, Malkasian D, Kelly DF: Endonasal versus supraorbital keyhole removal of craniopharyngiomas and tuberculum sellae meningiomas. Neurosurgery 64 (5 Suppl 2):269-286, 2009

13. Gardner PA, Kassam AB, Snyderman CH, Carrau RL, Mintz $\mathrm{AH}$, Grahovac S, et al: Outcomes following endoscopic, expanded endonasal resection of suprasellar craniopharyngiomas: a case series. J Neurosurg 109:6-16, 2008

14. Gardner PA, Prevedello DM, Kassam AB, Snyderman CH, Carrau RL, Mintz AH: The evolution of the endonasal approach for craniopharyngiomas. Historical vignette. J Neurosurg 108:1043-1047, 2008

15. Kasowski HJ, Nahed BV, Piepmeier JM: Transcallosal transchoroidal approach to tumors of the third ventricle. Neurosurgery 57 (4 Suppl):361-366, 2005

16. Kassam A, Snyderman CH, Mintz A, Gardner P, Carrau RL: Expanded endonasal approach: the rostrocaudal axis. Part I. Crista galli to the sella turcica. Neurosurg Focus 19(1):E3, 2005

17. Kassam A, Thomas AJ, Snyderman C, Carrau R, Gardner P, Mintz A, et al: Fully endoscopic expanded endonasal approach treating skull base lesions in pediatric patients. J Neurosurg 106 (2 Suppl):75-86, 2007

18. Kassam AB, Gardner PA, Snyderman CH, Carrau RL, Mintz AH, Prevedello DM: Expanded endonasal approach, a fully endoscopic transnasal approach for the resection of midline suprasellar craniopharyngiomas: a new classification based on the infundibulum. J Neurosurg 108:715-728, 2008

19. Kassam AB, Prevedello DM, Carrau RL, Snyderman CH, Thomas A, Gardner P, et al: Endoscopic endonasal skull base surgery: analysis of complications in the authors' initial 800 patients. A review. J Neurosurg 114:1544-1568, 2011

20. Kassam AB, Prevedello DM, Thomas A, Gardner P, Mintz A, Snyderman C, et al: Endoscopic endonasal pituitary transposition for a transdorsum sellae approach to the interpeduncular cistern. Neurosurgery 62 (3 Suppl 1):57-74, 2008

21. Kassam AB, Thomas A, Carrau RL, Snyderman CH, Vescan A, Prevedello D, et al: Endoscopic reconstruction of the cranial base using a pedicled nasoseptal flap. Neurosurgery 63 (1 Suppl 1):ONS44-ONS53, 2008

22. Katz S, Ford AB, Moskowitz RW, Jackson BA, Jaffe MW:
Studies of illness in the aged. The Index of ADL: a standardized measure of biological and psychosocial function. JAMA 185:914-919, 1963

23. Laws ER, Kanter AS, Jane JA Jr, Dumont AS: Extended transsphenoidal approach. J Neurosurg 102:825-828, 2005

24. Laws ER Jr: Transsphenoidal removal of craniopharyngioma. Pediatr Neurosurg 21 (Suppl 1):57-63, 1994

25. Sainte-Rose C, Puget S, Wray A, Zerah M, Grill J, Brauner R, et al: Craniopharyngioma: the pendulum of surgical management. Childs Nerv Syst 21:691-695, 2005

26. Samii M, Bini W: Surgical treatment of craniopharyngiomas. Zentralbl Neurochir 52:17-23, 1991

27. Samii M, Samii A: Surgical management of craniopharyngiomas, in Schmidek HH, Sweet WH (eds): Schmidek \& Sweet Operative Neurosurgical Techniques: Indications, Methods and Results, ed 4. Philadelphia: WB Saunders, 2000, Vol 1, pp 489-502

28. Schwartz TH: An eyebrow for an eyebrow and a nose for a nose. World Neurosurg [epub ahead of print], 2013

29. Van Gompel JJ, Frank G, Pasquini E, Zoli M, Hoover J, Lanzino G: Expanded endonasal endoscopic resection of anterior fossa meningiomas: report of 13 cases and meta-analysis of the literature. Neurosurg Focus 30(5):E15, 2011

30. Wilson DA, Duong H, Teo C, Kelly DF: The supraorbital endoscopic approach for tumors. World Neurosurg [epub ahead of print], 2013

31. Yaşargil MG, Abdulrauf SI: Surgery of intraventricular tumors. Neurosurgery 62 (6 Suppl 3):1029-1041, 2008

Manuscript submitted June 14, 2014.

Accepted July 16, 2014.

Portions of this work were presented in abstract as proceedings at the 6th World Congress for Endoscopic Surgery of the Brain, Skull Base \& Spine in Milan, Italy, on April 17, 2014, and have been accepted for presentation at the 15th European Associations of Neurosurgical Societies Congress in Prague, Czech Republic, on October 12, 2014.

Please include this information when citing this paper: DOI: 10.3171/2014.7.FOCUS14317.

Address correspondence to: Matteo Zoli, M.D., Centre of Pituitary Tumours and Skull Base Endoscopic Surgery, IRCCS Scienze Neurologiche, Via Altura 3, Bologna 40139, Italy. email: matteozeta @libero.it. 